\title{
Long-Term X-ray Variability in GX 354-0
}

\author{
A. K. H. Kong ${ }^{1}$, P. A. Charles ${ }^{2}$ and E. Kuulkers ${ }^{3}$ \\ Department of Astrophysics, Nuclear \& Astrophysics Laboratory, Keble Road, \\ Oxford OX1 3RH, U.K.
}

\begin{abstract}
We report for the first time the detection of long-term X-ray variability in the bright bulge source GX 354-0 (=4U 1728-34) observed with the All Sky Monitor (ASM) on board the Rossi X-Ray Timing Explorer (RXTE). The 2-year RXTE ASM database reveals significant power at $\sim 72$ days. Similar behaviour was seen in the 6 -year Ariel 5 ASM database, but at a period of $\sim 63$ days. The timescales and light curves resemble the $\sim 78$ days modulation seen in Cyg $\mathrm{X}-2$ and we therefore interpret this modulation in GX 354-0 as a super-orbital effect.
\end{abstract}

Key words: binaries: close, stars: individual: (GX 354-0, 4U 1728-34), stars: neutron, X-rays: stars

PACS: 97.60.J, 97.80.J

\section{Introduction}

The low-mass X-ray binary (LMXB) GX 354-0 (=4U 1728-34) is a well-known X-ray burster (e.g. Basinska et al. 1984) which has been classified as an atoll source based on EXOSAT data (Hasinger \& van der Klis 1989). Despite its detection in both soft and hard X-ray bands, its orbital period is still unknown. Based on Einstein HRI and infrared observations, Grindlay \& Hertz (1981) claimed the association of GX 354-0 with a heavily reddened globular cluster. However, the existence of this cluster was not confirmed by later infrared observations (van Paradijs \& Isaacman 1989). Apart from the bursting activities, GX 354-0 also exhibits complex behaviour on short time scales. Quasi-periodic oscillations (QPOs) at $363 \mathrm{~Hz}(2.75 \mathrm{~ms})$ during the burst itself were recently

1 E-mail: albertk@astro.ox.ac.uk

2 E-mail: pac@astro.ox.ac.uk

3 E-mail: erik@astro.ox.ac.uk 
discovered by the Rossi X-ray Timing Explorer (RXTE) (Strohmayer, Zhang \& Swank 1996; Strohmayer et al. 1996a; 1996b) providing the first evidence for a millisecond spin period in LMXB. A comprehensive discussion of these temporal and spectral characteristics can be found in Strohmayer et al. (1997). GX 354-0 is likely to be a typical LMXB and can be considered as a burster involving a rapidly rotating neutron star.

However, with no optical or infrared counterpart having yet been determined, because of the high extinction in this direction as well as the presumably large distance, there are few constraints on the nature or evolutionary state of GX 354-0. We therefore decided to exploit the long-term monitoring capabilities of the ASM on both the Ariel 5 and RXTE satellites in order to study the $\mathrm{X}$-ray behaviour of this source on timescales of weeks to months. In this way we could search for long-term (super-orbital) periods similar to those seen in other bright LMXBs, such as Cyg X-2 and X1820-30 (e.g. Smale \& Lochner 1992), and which might be related to either the accretion geometry, disc or neutron star properties.

\section{Observations}

\subsection{RXTE All Sky Monitor}

The All Sky Monitor (ASM) (Levine et al. 1996) on board the RXTE (Bradt, Rothschild \& Swank 1993) consists of three wide-angle shadow cameras equipped with proportional counters that provide regular and almost continuous intensity measurements of most bright X-ray sources in three different energy bands (1.5-3, 3-5 and 5-12 keV). The time resolution allows the ASM to cover $80 \%$ of the sky every 90 minutes. Combined with a $40 \%$ duty cycle (Levine et al. 1996), any given source is usually scanned 5-10 times per day. Both individual dwell and one-day average light curves are made available via the WWW by

the RXTE ASM team. Here, we use the dwell data in studying the detailed long-term light curve of GX 354-0.

In this analysis, we used 6550 measurements of GX 354-0 which were made by the RXTE ASM from 1996 February to 1998 February (see Fig. 1a).

\subsection{Ariel 5}

The Ariel 5 ASM experiment (Holt 1976) consisted of a pair of X-ray pinhole cameras with position sensitive proportional counters $(3-6 \mathrm{keV})$ that covered 
$75 \%$ of the sky during each orbit ( $\sim 100$ mins). From the archival HEASARC Ariel 5 database, we obtained 2142 data points on GX 354-0 spanning the period from October 1974 to March 1980 (see Fig. 2a).

\section{Analysis and Results}

The RXTE ASM light curve of GX 354-0 (Fig. 1a) shows substantial flaring activity, with count rates peaking at $\sim 26 \mathrm{ASM}$ counts $\mathrm{s}^{-1}$. In order to search for periodic phenomena in the data set, we used two different methods: the Lomb-Scargle periodogram (Lomb 1976; Scargle 1982) and phase dispersion minimization (PDM; Stellingwerf 1978). The Lomb-Scargle periodogram is a modification of the discrete fourier transform which is generalized to the case of uneven spacing. However, it is most sensitive in those cases where any modulation present is sinusoidal. PDM is suitable even where the data is nonsinusoidal and works by dividing the data into phase bins and minimizing the dispersion within the bins. The deepest minimum relates to the period of the strongest modulation.

By applying the Lomb-Scargle periodogram to the RXTE ASM data, a distinct peak is found at 71.7 days. We determined the uncertainties on this period by generating 10,000 artificial data sets with the same variance, amplitude and period as the true data. The distribution in the resulting peaks leads to an error in the true period of $\sim 0.5 \mathrm{~d}(1 \sigma)$. In Fig. $1 \mathrm{~b}$ (upper panel) we plotted the 99\% significance level. This is determined by generating noise data sets with the same time intervals and variance as the true data and then performing the Lomb-Scargle periodogram on the resulting data sets. The peak power in each periodogram (which must be purely due to noise) was then recorded. This was also repeated 10,000 times for good statistics. We conclude that our peak at 71.7 days is highly significant. This result is also independently confirmed by the PDM analysis (Fig. 1b, lower panel). We note, however, that the deepest minimum occurs at $\sim 117$ days, but this is due to observation gaps in the RXTE ASM light curve (Fig. 1a). The folded light curve of our RXTE ASM data on the period of 71.7 days is shown in Fig. 1c; phase zero is defined by the first data point.

The Ariel 5 ASM data do not show the variation as clearly as the RXTE ASM data, but some modulation can be discerned. We note that there are various data gaps which will affect the window function. Both the Lomb-Scargle periodogram and PDM (Fig. 2b, upper and middle panels) show a peak near 365 days, which is due to the yearly variation in the count rate from solar X-ray scattering or fluorescing from the Earth's atmosphere (Priedhorsky, Terrell \& Holt 1983). The second strongest peak is at $63 \pm 0.1$ days (1- $\sigma$ level) and lies well above the $99 \%$ confidence level. The window function is also shown (Fig. 
$2 \mathrm{~b}$, lower panel) to indicate the alias periods that would be induced by the yearly variation and observation gaps. Fig. 2c shows the folded light curve of the Ariel 5 ASM data on the period of 63 days, where phase zero is defined by the first data point.

We also analysed the data from the Vela 5B XC (Conner, Evans \& Belian 1969) which monitored the sky from 1969 May to 1979 June in the 3-12 keV band. However, due to the low sensitivity of this instrument, no variability was detectable.

\section{Discussion}

We have detected a $71.7 \pm 0.5 \mathrm{~d}$ periodicity in the $R X T E$ ASM light curve and a $63 \pm 0.1 \mathrm{~d}$ periodicity in the Ariel 5 ASM light curve of the LMXB GX 354-0. Such a long period modulation has hitherto been rather uncommon in LMXB. From the long-term variability survey carried out by Smale \& Lochner (1992) using Vela 5B, only 3 out of 16 LMXBs (X1820-30, X1916-05, Cyg X-2) were found to have long-term periods. In particular, X1820-30 and X1916-05 have periods of 176 days and 199 days, respectively, while recent observations by RXTE ASM confirm the long-term period of $\sim 78$ days in Cyg $\mathrm{X}-2$ (Wijnands et al. 1996). These long-term periods are designated superorbital, as the orbital periods are known for all three objects (11.3 mins, 50 mins and $9.8 \mathrm{~d}$, respectively). In massive X-ray binaries, super-orbital periods are more common. Cyg X-1, SS433, LMC X-4, Her X-1 and several other sources all have super-orbital periods in the range of 30-300 days (e.g. Priedhorsky $\&$ Holt 1987). The cause of these super-orbital periods is still a subject of debate, and possible explanations include the precession of a tilted accretion disk, neutron star precession, mass transfer feedback and triple systems (see Priedhorsky \& Holt 1987 and Schwarzenberg-Czerny 1992 for more details).

In the case of LMXBs the super-orbital period is much more rare and there is a large spread in values of the ratio of super-orbital to orbital periods. For $\mathrm{X} 1820-30, \mathrm{X} 1916-05$ and $\mathrm{Cyg} \mathrm{X}-2$, the ratio is 22100, 5750 and 8 respectively, whereas in massive systems it is in the range of 10 to 100 (see e.g. Wijers \& Pringle 1998). The long-term variation in LMXBs may instead be due to radiation driven warped accretion discs (e.g. Wijers \& Pringle 1998) or a disc instability in the system (Priedhorsky \& Holt 1987; Dubus et al. 1998).

Many orbital periods of bright galactic sources (including GX 354-0) remain unknown mainly due to the heavy optical extinction and/or crowded regions. While the known orbital periods of LMXBs range from 11 mins to $\sim 10 \mathrm{~d}$, Cyg X-2 and X0921-63 actually have long orbital periods ( $\sim 10$ days). We note that the RXTE ASM light curves of Cyg X-2 (Fig. 3a) and GX 354-0 
are rather similar. This similarity is enhanced by our analysis of the much more extensive $R X T E$ ASM database of Cyg $\mathrm{X}-2$ that is available now. The LombScargle periodogram shows a $69 \pm 0.4 \mathrm{~d}$ period (Fig. 3b) in addition to the already noted $\sim 78 \mathrm{~d}$ period (Wijnands et al. 1996). Fig. 3c shows the folded light curve of Cyg X-2 with a period of 69 days, where phase zero is defined by the first data point. A recent study of GX $1+4$ by Chakrabarty \& Roche (1997) has suggested that the orbital period of this source may exceed 100 days or even 260 days. However, since any orbital modulation should be uniquely stable, we conclude that our periods of $63 \mathrm{~d}$ and $72 \mathrm{~d}$ found for GX 354-0 are "super-orbital" and not orbital. Further optical/infrared campaigns are needed to reveal its orbital period and to make further progress in this area.

\section{Acknowledgements}

We are grateful to Guillaume Dubus for useful comments on the discussion section. This paper utilizies quick-look results provided by the $A S M / R X T E$ team and data obtained through the HEASARC Online Service of NASA/GSFC.

\section{References}

[1] Basinska E. M., Lewin W. H. G., Sztajno M., Cominsky L. R., Marshall F. J., 1984, ApJ, 281, 337.

[2] Bradt H. V., Rothschild R. E., Swank J. H., 1993, A\&AS, 97, 355.

[3] Chakrabarty D., Roche P., 1997, ApJ, 489, 254.

[4] Conner J. P., Evans W. D., Belian R. D., 1969, ApJ, 157, L157.

[5] Dubus G. et al., 1998, in preparation.

[6] Grindlay J. E., Hertz P., 1981, ApJ, 247, L17.

[7] Hasinger G., van der Klis M., 1989, A\&A, 225, 79.

[8] Holt S. S., 1976, Ap\&SS, 42, 123.

[9] Levine A. M., Bradt, H. V., Cui W., Jernigan J. G., Morgan E. H., Remillard

R., Shirley R. E., Smith D. A., 1996, ApJ, 469, L33.

[10] Lomb N. R., 1976, Ap\&SS, 39, 447.

[11] Priedhorsky W .C., Terrel J., Holt S. S., 1983, ApJ, 270, 233.

[12] Priedhorsky W. C., Holt S. S., 1987, SSR, 45, 291.

[13] Smale A. P., Lochner J. C., 1992, ApJ, 395, 582. 
[14] Scargle J. D., 1982, ApJ, 263, 835.

[15] Schwarzenberg-Crerny A., 1992, A\&A, 289, 149.

[16] Stellingwerf R. F., 1978, ApJ, 224, 953.

[17] Strohmayer T. E., Zhang W., Swank J. H., 1996, IAU Circ. 6320.

[18] Strohmayer T. E., Zhang W., Smale A., Day C., Swank J. H., Titarchuk L., Lee U., 1996a, IAU Circ. 6387.

[19] Strohmayer T. E., Zhang W., Swank J. H., Smale A., Titarchuk L., Day C., 1996b, ApJ, 469, L9.

[20] Strohmayer T. E., Zhang W., Swank J. H., 1997, ApJ, 487, L77.

[21] van Paradijs J., Isaacman R., 1989, A\&A, 222, 129.

[22] Wijers R. A. M., Pringle J. E., 1998, MNRAS, submitted.

[23] Wijnands R. A. D., Kuulkers E., Smale A. P., 1996, ApJ, 473, L45. 

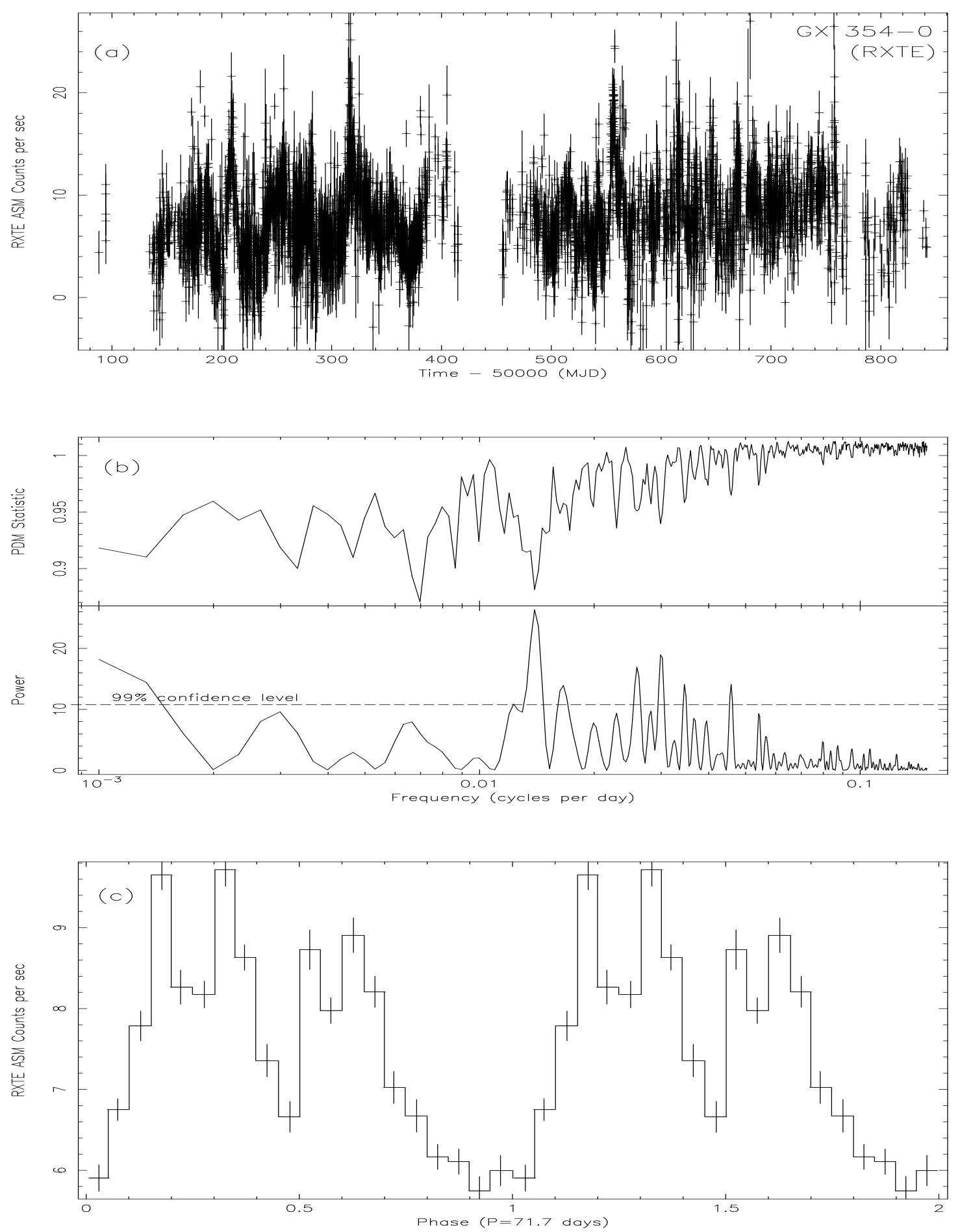

Fig. 1. a) RXTE ASM 1.5-12 keV light curve of GX 354-0 from 1996 February to 1998 February. b) PDM analysis (upper panel) of the RXTE ASM data, with the deepest minimum at $\sim 117$ days and the next deepest at $\sim 72$ days; the Lomb-Scargle periodogram (lower panel) of the same data with a peak at the same 72 day period. The $99 \%$ confidence level is shown by a dashed line. c) RXTE ASM folded light curve of GX 354-0 on a period of 71.7 days. Two cycles are shown for clarity. Phase zero is arbitrary set at the time of the first data point (JD2450088.6) 

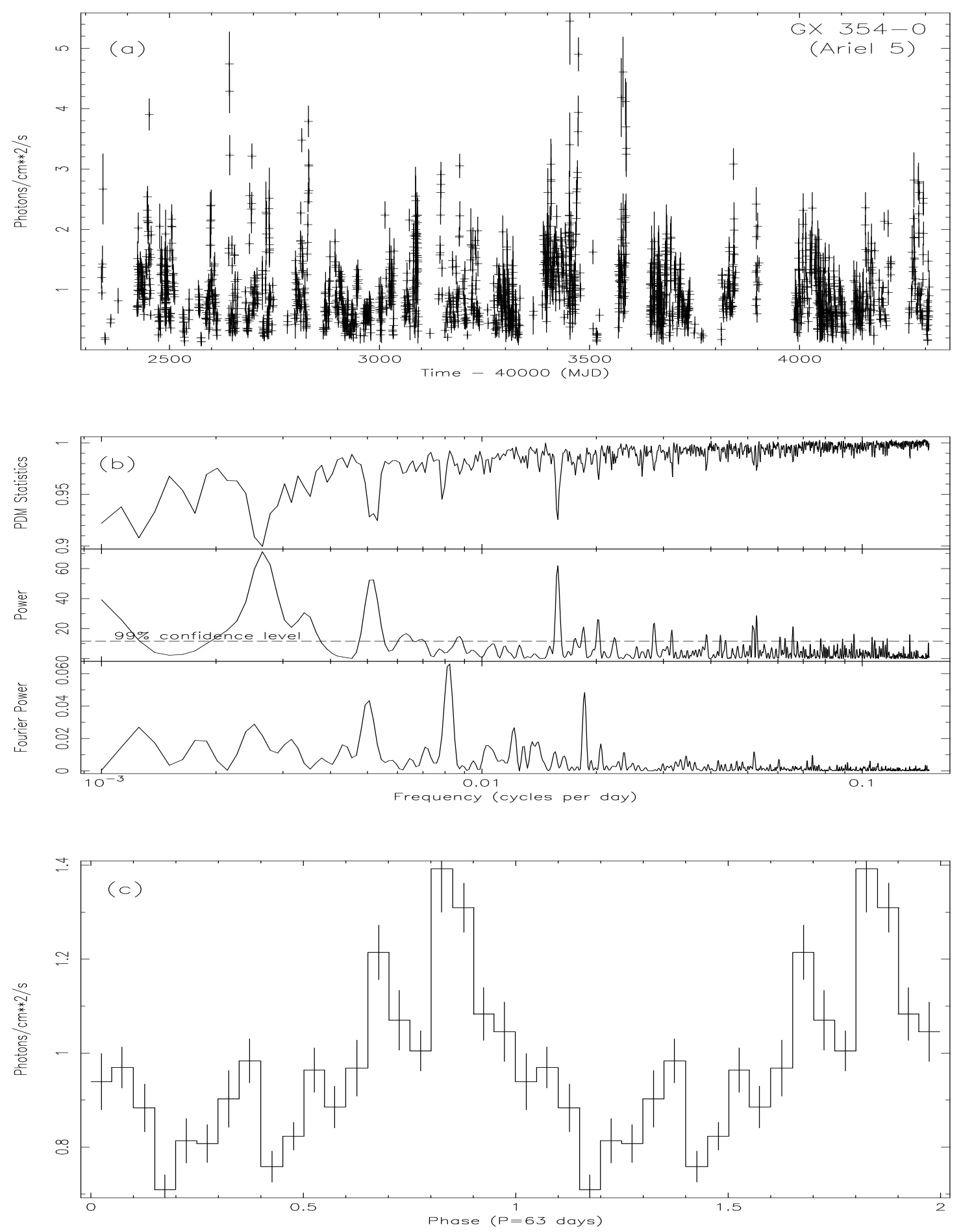

Fig. 2. a) Ariel 5 ASM 3-6 keV light curve of GX 354-0 from 1974 October to 1980 March. b) PDM analysis of the Ariel 5 ASM data (upper panel), with the deepest minimum at $\sim 365$ days (due to solar scattering, see text) and the second deepest at $\sim 63$ days; the Lomb-Scargle periodogram (middle panel) of the same data showing the same peak as the PDM at $~ 63$ days. The $99 \%$ confidence level is shown as a dashed line. The window function of the Ariel 5 ASM data (lower panel) clearly shows the alias peak in the two periodograms which are due to the observation gaps. c) Ariel 5 ASM folded light curve of GX $354-0$ on a period of 63 days. Two cycles are shown for clarity. Phase zero is arbitrary set at the time of the first data point (JD2442338.6) 

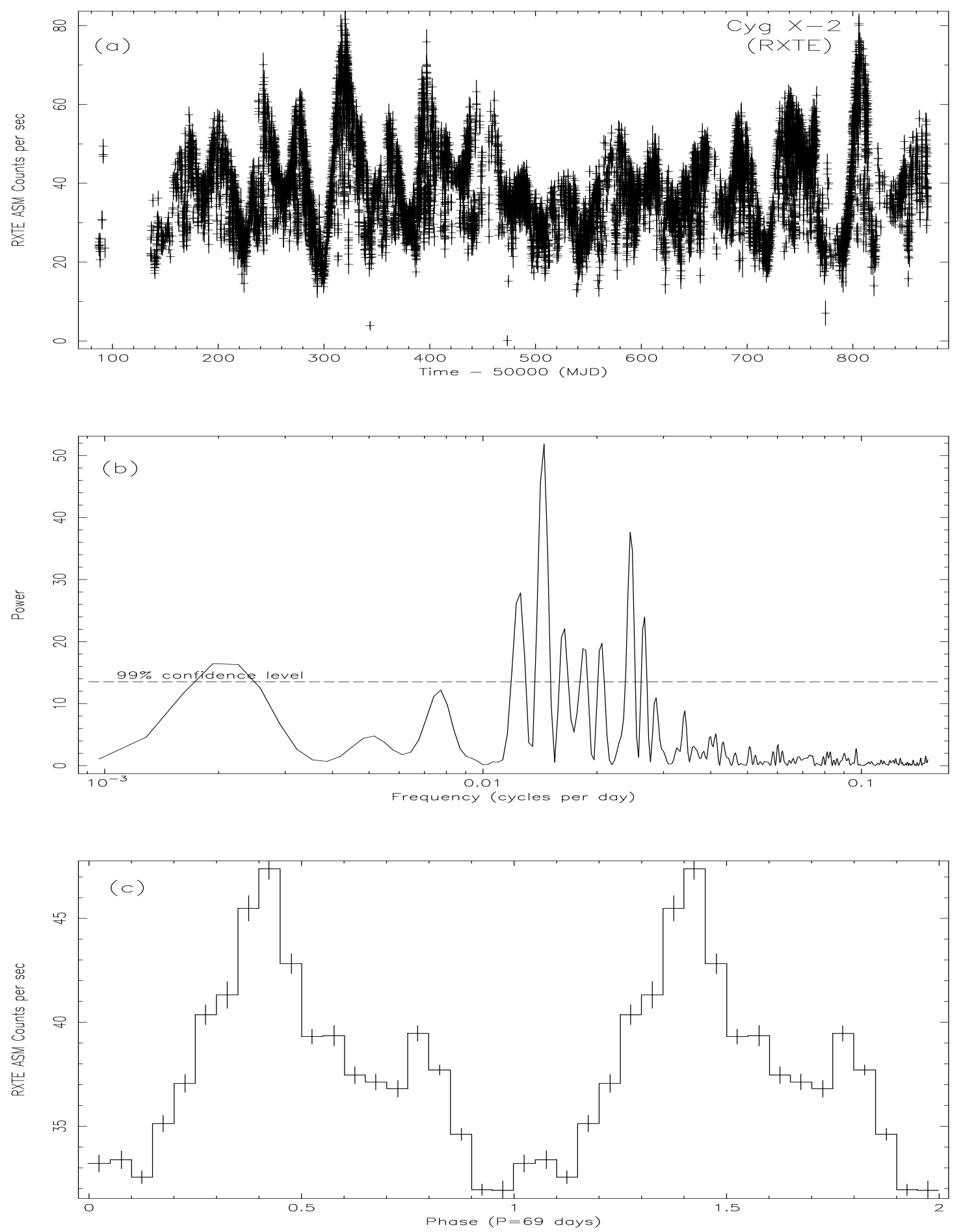

Fig. 3. a) RXTE ASM light curve of Cyg X-2 from 1996 February to 1998 February. b) Lomb-Scargle periodogram of the $R X T E$ ASM data on Cyg X-2 showing a strong peak at $\sim 69$ days. The $99 \%$ confidence level is shown for reference. c) Folded light curve of the RXTE ASM data of Cyg $\mathrm{X}-2$ with period 69 days. Phase zero is arbitrary set at the time of the first data point (JD2450087.8) 